\title{
Non-suicidal self-injury in a sample of university students in Tehran, Iran: prevalence, characteristics and risk factors
}

\section{ABSTRACT}

Despite increased empirical and clinical attention to non-suicidal self- injury (NSSI) in Western countries, far less is known about NSSI in non- Western cultures. This study is the first to investigate the prevalence, characteristics and risk factors of NSSI in a sample of university students in Tehran, Iran. All participants $(n=554$, mean age $=$ $22.65,57.2 \%$ female) were asked to self-report on NSSI over their lifetime. The Difficulties in Emotion Regulation Scale, Depression Anxiety Stress Scales, and Suicidal Behaviors Questionnaire-Revised were employed. A lifetime NSSI prevalence of 12.3\% $(n=68)$ was found with no gender differences. Using logistic regression, lack of emotional awareness remained negatively significant for females after controling for anxiety, depression and suicidality; however, after controlling for the psychological symptoms, no relations were found between emotion dysregulation and a history of lifetime NSSI for male students. In a separate logistic regression, lifetime NSSI predicted suicide attempt above and beyond depressive symptoms and suicidal ideation across gender. Our results on the relations between emotion dysregulation and lifetime NSSI contradict the ample research in the West, emphasizing emotion dysregulation as a risk factor of NSSI and suggest that this relation might vary across cultures. The results are situated within the sociocultural context and compared and contrasted with Western data.

\section{Introduction}

Non-suicidal self-injury (NSSI) is defined as the deliberate and immediate destruction of one's own body tissue without conscious suicidal intent and for reasons not 
socially accepted (Nixon \& Heath, 2009). Methods of NSSI include, but are not limited to, cutting, scratching, burning, head banging and self-hitting (Nixon \& Heath, 2009). Non-suicidal self-injury is a serious public health problem that can result in adverse physical consequences and is associated with shame, social isolation and poor academic achievement (Bentley, Nock, \& Barlow, 2014). Although NSSI and suicidal behaviors are considered different constructs (Butler \& Malone, 2013), NSSI is strongly linked to suicide attempts (SA) and self-inflicted death among young individuals (Klonsky, May, \& Glenn, 2013). Currently, NSSI is clearly described in Section III of the Diagnostic and Statistical Manual of Mental Disorders - 5th Edition (DSM-5, American Psychiatric Association, 2013) as a separate clinical con- dition requiring further research.

Non-suicidal self-injury is common among university students, with an approximate lifetime prevalence rate of $10.5 \%$ ranging from 3.8 to $17.3 \%$, mostly obtained from Western samples (Swannell, Martin, Page, Hasking, \& St John, 2014). Although results on gender differences of NSSI prevalence rates among university students are inconsistent, most of the studies report gender differences in preferred NSSI method; for instance, females are more likely to engage in scratching, cutting and wound interference, and males are more likely to engage in punching, head banging and burning (Andover, Primack, Gibb, \& Pepper, 2010; Bresin \& Schoenleber, 2015; Whitlock, Eckenrode, \& Silverman, 2006, 2011). The reported average age of NSSI onset for the majority of individuals appears to be 14 to 16 years old, with a decrease in reported NSSI from early to middle adulthood (Moran et al., 2012; Plener, Schumacher, Munz, \& Groschwitz, 2015).

Individuals who engage in NSSI tend to experience high levels of hyperarousal to distress and tend to have difficulties in regulating their inner states (Nock, 2010); 
therefore, NSSI episodes usually follow negative emotional/cognitive states and may work to regulate the intolerable emotional/cognitive state (Nock, 2010). In addition, research indicates that negative states such as depressive symptoms, loneliness, anxiety, dissociation symptoms and suicidal ideations are strongly associated with NSSI (Glenn \& Klonsky, 2011; Gratz, Conrad, \& Roemer, 2002; Plener et al., 2015; Wilcox et al., 2012). There is ample research emphasizing the role of emotion dysregulation as one of the most important risk factors of NSSI, even after controlling for psychopathology and other risk factors of NSSI (Adrian, Zeman, Erdley, Lisa, \& Sim, 2011; Gratz \& Roemer, 2008). In fact, it is documented that emotion regulation is the most important function of NSSI (Andover \& Morris, 2014; Klonsky, 2007). Gratz and Roemer (2004) have introduced a multidimensional notion of emotion regulation that considers the functionality of emotions and emphasizes modulating the urgency and duration of emotions instead of controlling or eliminating them. According to Gratz and Roemer (2004), there are six main domains in regulating emotions: (1) awareness of emotions, (2) clarity of emotions, (3) acceptance of emotional experience, (4) the ability in engaging in goal-directed behaviors, (5) the ability to inhibit impulsive behaviors and, finally, (6) accessing strategies to effectively regulate emotions. Research supports the strong association between the multidimensional conceptualization of emotion dysregulation and NSSI, notably the difficulty in accessing effective strategies (Andover \& Morris, 2014).

Emerging non-Western data on NSSI suggests that cultural factors may have a great impact on the meanings, risk factors and the gender differences in NSSI (Gholamrezaei, De Stefano, \& Heath, 2015). Specifically, when compared with Western 
data, results of studies conducted in non-Western countries do not consistently support the emotion regulation functionality of NSSI, and a more interpersonal functionality of NSSI in collectivistic societies can be hypothesized (Gholamrezaei et al., 2015). For instance, in a longitudinal study among Chinese adolescents, You, Leung and $\mathrm{Fu}(\underline{2012})$ found that: (1) intense and unstable interpersonal relationship patterns predicted NSSI over time and (2) engaging in NSSI led to elevated negative emotions over time, but not vice versa. Additionally, cultures might encourage specific types of self-injury that have certain religious and/or cultural purposes or motivations, while prohibiting and stigmatizing similar forms of self-injury conducted outside of religious/cultural contexts (Favazza, 1996). For example, despite the clear prohibition of suicidal and self-injury by Islam, a minority of Shiite Muslims, most of whom live in Iran and several other Middle Eastern countries, mourned the martyrdom of the last descendant of Mohammed by engaging in specific self-injurious rituals (Baasher, 2001). The influence of cultural factors in self-injurious behaviors is so profound that self-injurious behaviors for culturally sanctioned purposes (e.g., religious rituals) are explicitly excluded from the definition of NSSI in the research literature as well as DSM-5 criteria of potential NSSI disorder (American Psychiatric Association, 2013). Considering the interaction of sociocultural factors and self-harming behaviors, more research of non-Wes- tern samples is crucial to contextualize our understanding of NSSI.

Iran is a Middle Eastern country with a specific sociopolitical situation and a young population. Research suggests particular patterns of suicidal behaviors among young individuals, notably young women, in Iran. For instance, studies have documented that Western women usually avoid using suicide methods that can lead to facial 
disfiguration such as shooting themselves in the head (Stack \& Wasserman, 2009). Meanwhile, the typical method of suicide by women in western Iran is self-burning, which leads to death or serious and permanent injuries and disfiguration (Panaghi et al., 2007). Furthermore, research suggests that unlike in the West, the majority of people who attempt or commit suicide in Iran do not have a history of psychiatric disorder or alcohol use, but they are more likely to have been assaulted physically or verbally (Panaghi, Ahmadabadi, Peiravi, \& Zahra Abolmasoomi, 2010; Sheikholeslami, Kani, \& Ziaee, 2008). Moreover, it seems that married women attempt suicide more than women who are single, recently separated, divorced or widowed (Sheikholeslami et al., 2008). This finding is inconsistent with studies conducted in the West, which suggest that marriage is a protective factor for women (Corcoran \& Nagar, 2010) or that there is no difference in suicide risk between divorced and married women (Kposowa, 2000).

In summary, there seems to be different pathways for various aspects of suicidal behaviors in Iran, which are most likely influenced by the sociocultural factors at play. Considering the close relation- ship between suicidal behaviors and NSSI, studying nonsuicidal self-injury in Iran might reveal different patterns in how NSSI manifests in the context of a non-Western culture. The goal of this study is three-fold. The first objective of this study is to investigate the prevalence and characteristics of NSSI among a sample of university students in Tehran. The second objective is to investigate whether emotion dysregulation will remain an important risk factor for NSSI after controlling for associated psychological symptoms of anxiety, depression, suicidality and stress. Gender differences have remained unclear in NSSI prevalence and risk factors in non-Western countries (Gholamrezaei et al., 2015); therefore, separate analyses were conducted for 
male and female students. Finally, the third objective is to investigate the relationship between NSSI and SA after controlling for depressive symptoms and suicide ideations.

\section{Methods}

\section{Participants and procedures}

The participants in this study were 556 students $(57.2 \%$ female; mean age $=$ $22.65, S D=3.45)$ selected using a convenience sampling method. Following the approval from related boards and ethical committees, the sample was recruited from a large public university in Tehran with approximately 17,500 undergraduate and graduate students. The sample was recruited in mostly undergraduate classrooms from different faculties. Table 1 summarizes some demographic characteristics of the sample.

Various classes across different faculties were visited to request participation in the study. Under- graduate classes had a capacity of 35 to 60 students. The project was introduced as a study on 'stress and coping among SBU students' and the procedure of the study was also explained. Students were informed that participation was voluntary and they could withdraw from the survey without any penalty or prejudice. Study packages containing a series of anonymous self-report questionnaires were given to students who agreed to participate in the study. In addition to the questionnaires, a contact information sheet indicating available on-campus and community mental health resources were provided to all the students. Approximately $70 \%$ of contacted students participated in the study.

\section{Measures}

\section{Difficulties in Emotion Regulation Scale (DERS)}

The DERS (Gratz \& Roemer, 2004) is a self-report questionnaire that consists of 
36 items rated on a five-point Likert scale ranging from 1 to 5 , where 1 is 'almost never' and 5 is 'almost always'. The DERS assesses difficulties in different aspects of emotion regulation and consists of six main difficulties: (1) lack of emotional awareness (aware), (2) lack of emotional clarity (clarity), (3) non-acceptance of emotional responses (nonaccept), (4) limited access to emotion regulation strategies (strategies), (5) impulse control difficulties (impulse) and (6) difficulties engaging in goal-directed behavior (goals). The DERS has high internal consistency (Cronbach's $\alpha=0.93$ ), good test-retest reliability $(=.88, p<.01)$ and adequate construct and predictive validity (Gratz \& Roemer, 2004). The internal consistency of the Farsi version of the DERS ranges from .66 to .88 , and a one-week test- retest reliability coefficient ranges from .79 to .91 across subscales (Khanzadeh, Saeediyan, Hosseinchari, \& Edrissi, 2012). The Farsi version of DERS was used in the present study. In the present study, internal consistency measured by Cronbach's alpha was .91 .

\section{Non-Suicidal Self-Injury Scale}

This questionnaire was mostly adopted from the NSSI follow-up section of the How I Deal with Stress measure (HIDS; Ross \& Heath, 2007). Some items from the Inventory of Statements About Self-Injury (ISAS) Section I (Klonsky \& Glenn, 2009) were added as well. The following sections were adopted from the HIDS: (1) NSSI methods, including self-cutting, self-burning, scratching, head banging and self-hitting. Self-biting and punching a wall were also added to the list; (2) a sec- tion assessing the emotions felt after an episode of NSSI was included. Participants were able to select multiple emotions on the list; (3) items in the HIDS questionnaire assessing other NSSI character- istics, including age of onset, three-month NSSI prevalent and frequency of the 
behaviour, were included as well. The following items were adopted from the ISAS: (1) an item assessing presence of pain while self-injuring, (2) an item regarding the desire to stop NSSI and, finally, (3) an item assessing if engagement in NSSI was in the presence of others or alone.

The questionnaire was translated into Farsi and back translated. There was little difference between the original version of the questionnaire and the back-translated English version. The instrument started with an introduction providing a definition of NSSI (i.e., some young individuals self-injure to deal with personal or social/interpersonal distress and life problems. Self-injury is defined as behaviors that lead to body tissue damage without wanting to die. Behaviors such as cut- ting or headbanging that result in bleeding, bruising, or any mark without any suicidal intent. Please read the following question and if your response is in the affirmative continue filling out this section, if your answer is no, please skip to the next section), followed by a screening question, 'Have you ever self-injured even once in your life?'

\section{Depression Anxiety Stress Scales (DASS-21)}

The short version of the DASS consists of 21 items rated on a four-point Likert scale and assesses negative affectivity over the previous week. The DASS-21 is composed of three subscales measuring depression, anxiety and stress (Lovibond \& Lovibond, 1995). Evidence suggests strong construct validity and good internal consistency, ranging from .89 to .90 across subscales, for the DASS-21 among nonclinical samples (Henry \& Crawford, 2005). The Farsi version of the DASS-21 is one of the most widely used measures among both clinical and non-clinical Iranian samples and has shown excellent psychometric properties, including strong internal consistency 
(anxiety, $\alpha=.88$; depression, $\alpha=.92$; and stress, $\alpha=.82$ ), high four-week test-retest reliability $(r=.72)$ and strong construct validity (Bayani, 2010). In the current study, internal consistency measured by Cronbach's alpha was .93 .

\section{The Suicidal Behaviors Questionnaire-Revised (SBQ-R)}

Developed by Osman et al. (2001), this instrument consists of four items assessing lifetime SA, 12-month SA, threats of suicidal behaviors and the likelihood of future SA. There are different scaling systems for each item. Research suggested good internal consistency $(\alpha=.83)$ in an under- graduate sample (Gutierrez, Osman, Barrios, \& Kopper, 2001), and strong construct validity in both clinical and community samples (Osman et al., 2001). The SBQ-R has been translated into Farsi and validated in an outpatient sample (Safa, Boroujerdi, Talischi, \& Masjedi, 2014); however, the psychometric properties of the validated Farsi version of the SBQ-R are not published. In the current study, the computed Cronbach's alpha was .85 .

\section{Statistical analyses}

To address the first research objective, descriptive analyses were used to determine NSSI prevalence and characteristics. T-tests and chi-squares were also conducted to examine gender differences in prevalence and characteristics of NSSI.

We examined the NSSI profile of participants who indicated once in their lifetime NSSI engagement $(n=9)$ before conducting the analyses to exclude participants who engaged in a minor NSSI incident once in their lives. According to their profile, there were indicators of underreporting for five of these participants (e.g., they were not sure if they had stopped the behavior or they indicated severe one-time self-injury such as cutting the face). The other four participants were removed from the analyses for the 
second and third objectives.

To investigate whether emotion dysregulation remains an important risk factor for NSSI after controlling for the psychological symptoms, hierarchical binary logistic regressions were conducted for male and female participants separately with likelihood of lifetime NSSI incidence as the dependent variable. To determine which variables can be entered in the logistic regression, associations of the predictors with NSSI status (i.e., having a history of NSSI or not) were examined (see Table 2). As shown in Table 2, stress, depressive symptoms and three DERS subscales (non-acceptance, clarity and aware), were not correlated with NSSI status for male students and were therefore not entered in the logistic regression model for male participants. The DERS subscales of goals and clarity were not significantly correlated with NSSI status for female students, and were therefore removed from the model for female participants. Although stress was significantly correlated with NSSI for female students, it was removed due to its high correlations with other DASS subscales of depression $(r p b=.76, \mathrm{p}<.001)$ and anxiety $(r p b=.75, p<.001)$.

To investigate the third objective, a second hierarchical binary logistic regression was conducted with SA prevalence as the dependent variable across gender. Depressive symptoms and suicide ideation were entered in the model as block 1, and a history of NSSI were entered as block 2. Some initial analyses were examined to investigate the association of SA to NSSI, last-year suicidal ideation and depressive symptoms separately for both genders (see Table 3).

\section{Results}

\section{Prevalence and characteristics of NSSI}


Table 4 demonstrates the prevalence rates of NSSI for the overall sample $(n=$ 554) and separately for male and female students. In the screening questionnaire, a total of $68(12.3 \%)$ students reported that they hurt themselves on purpose without suicidal intent over their lifetime. There were 28 participants (5.1\%) who indicated that they had engaged in NSSI within the last three months. Of those reporting a lifetime NSSI engagement, $65 \%(n=44)$ indicated engaging in multiple methods of NSSI. The frequency of self-injury assessed in the follow-up section of the questionnaire were as follows 9 students (1.6\%) had self-injured once, 23 students (4.2\%) 2-4 times, 13 students (2.3\%) 5-10 times, 8 students (1.4\%) 11-50 times, 10 students (1.8\%) 51-100 times and 5 students did not answer the question. The mean age of onset was 13.7 ( $S D=$ $5.15, M=15)$ years, with the majority of partici- pants (68.9\%) reporting their first incidence of NSSI before the age of 17 years. Of those endorsing any form of NSSI, 54\% indicated they had self-injured where they were not alone; however, it was not assessed in the presence of whom the self-injury was carried out. Of the $34 \%$ of students who reported any form of NSSI, they also indicated they did not feel pain when they selfinjured, $12 \%$ indicated they did not have the desire to stop NSSI and 32\% indicated that no one knew about their NSSI.

The most frequently reported methods used for male students were punching against a wall and cutting, followed by self-hitting. There was no significant difference between punching a wall and cutting for male students $(\mathrm{X} 2[1=3.49, \mathrm{p}=.062)$, but a trend towards a significant difference was found between frequencies of punching a wall and self-hitting $(\mathrm{X} 2[1]=3.75, p=.053)$. The most frequently reported methods for female students were punching against a wall and self-hitting, followed by cutting, with a 
significant difference between the reported frequencies of punching a wall and cut- ting $(\mathrm{X} 2[1]=4.88, p<.05)$. Frequencies of NSSI methods used are showed in $\underline{\text { Table } 4}$ separately for male and female students. The endorsed emotions felt after a NSSI episode were feeling calm (57.6\%), sad (45.5\%), angry (39.4\%), frustrated (39.4\%) and anxious (39.4\%) for male students. For female students, the emotions reported after an NSSI episode were feeling sad (57\%), frustrated (49\%), nervous (46\%), guilty (46\%) and calm (40\%). The least reported emotions were energetic (5\%) and happy (7\%) for both genders.

Chi-square analyses indicated that no gender differences were found regarding NSSI methods used, the presence of pain, desire to stop NSSI, and self-injuring in the presence of others. Further- more, no gender differences were found in the lifetime and three-month prevalence rates of NSSI $(\mathrm{X} 2[1]=1.166, p=.28$ and X2[1] $=1.403, p=$ .236 , respectively). In addition, t-test analyses indicated no gender differences in terms of NSSI frequency and age of onset $(t[61]=.77, p=.44$ and $t[59]=-.817, p=.417$, respectively).

\section{Risk factors for NSSI}

Hierarchical binary logistic regressions were conducted for male and female participants separately. The psychological symptoms were entered simultaneously in the first block, and the significantly correlated DERS subscales with NSSI were entered simultaneously in the second block.

Goodness-of-fit was examined using Hosmer-Lemeshow chi-square test, indicating an adequate goodness-of-fit for the full model for both male and female participants $\left(\mathrm{X}^{2}[8]=11.3, p=.18\right.$ and $\mathrm{X}^{2}[8]=.3 .38, p=.91$, respectively $)$. Results of the 
omnibus test showed that the full model was significant for both male and female participants $\left(\mathrm{X}^{2}[5]=19.01, p<.005\right.$ and $\mathrm{X} 2[7]=34.49, p<.001$, respectively $)$ indicating that the risk factors, as a set, reliably predict NSSI group membership. Altogether, the predictors accounted for 14\% (Nagelkerke R2) of variability in NSSI group member- ship for males and 21.9\% (Nagelkerke R2) of variability in NSSI group membership for females. As can be seen from Table 5, for male students, anxiety and suicide were significant predictors of NSSI in the first model. After entering the DERS subscales, only suicide ideation remained significant. No DERS subscale significantly predicted NSSI after controlling for anxiety and suicide ideation. For female students, suicide ideation was the only significant predictor in the first model. In the full model, after entering the DERS subscales, suicide ideation remained significant. Aware was the only DERS subscale that significantly and negatively predicted NSSI after controlling for anxiety, depression and suicide ideation among female students, meaning that being more aware of one's own emotional experience significantly predicted a history of NSSI after controlling for the other variables.

\section{The relation between NSSI and SA}

A total of 23 participants $(4.2 \% ; 12$ female, 11 male) reported SA in their lifetime. The gender difference in SA incidence was not statistically significant $\left(\mathrm{X}^{2}[1]=\right.$ $.266, p=.6)$.

Hierarchical binary logistic regressions were employed separately across genders. Depressive symptoms and suicidal ideation were entered simultaneously in the first model. In the second model, NSSI incidence was entered.

Goodness-of-fit was examined using Hosmer-Lemeshow chi-square test, 
indicating an adequate goodness-of-fit for the full model for both male and female participants $\left(\mathrm{X}^{2}[7]=4.09, p=.77\right.$ and $\mathrm{X}^{2}[8]=10.29, p=.245$, respectively). Results of the omnibus test showed that the full model was significant for both male and female students $\left(\mathrm{X}^{2}[3]=23.19, p<.001\right.$ and $\mathrm{X}^{2}[3]=23.66, p<.001$, respectively) suggesting that the risk factors, as a set, reliably predict SA group membership.

Altogether, suicide ideation, NSSI, and depressive symptoms accounted for $30 \%$ (Nagelkerke R2) of variability in SA group membership for males and 26\% (Nagelkerke R2) of variability in SA group membership for females. As can be seen from Table 6 , NSSI remained a significant predictor of SA after controlling for depressive symptoms and suicidal ideation for both male and female students.

\section{Discussion}

Investigating NSSI across different cultures contextualizes the field of study and suggests which factors may be influenced by culture and which factors may be more universal (Canino, Lewis- Fernandez, \& Bravo, 1997). The results of the current study suggest a $12.3 \%$ lifetime prevalence rate of NSSI among the university sample in Tehran, which reveals that NSSI is a widespread behavior among this population in Iran. This rate falls towards the lower end of Western estimates, which range between approximately 12 and 21\%; however, a screening question was used in this study to assess the prevalence of NSSI and research suggests that using checklists of NSSI methods identifies a higher rate of NSSI (Muehlenkamp, Claes, Havertape, \& Plener, 2012). Consistent with the Western data (Plener et al., 2015), the majority of the students in our sample with a NSSI history started self- injuring during their adolescence; however, the average age of onset reported in our sample (i.e., 13.7) appeared to be around two years younger than the 
average age of onset reported in Western university samples (e.g., Heath, Toste, Nedecheva, \& Charlebois, 2008; Whitlock et al., 2006, 2011). Younger age of NSSI onset is associated with severity of the condition in studies conducted in the West (Klonsky \& Olino, 2008); however, the earlier age of onset in our sample might be more related to cultural factors. Longitudinal studies are needed to confirm and explain the result.

Although cutting is reported as the most common method in Western samples, notably by females (Heath et al., 2008; Whitlock et al., 2011), interestingly, in our sample, the most frequently reported methods for male students were punching a wall and cutting and the most frequently reported methods for female students were punching a wall and self-hitting. Punching a wall and self-hitting are NSSI methods that are more utilized by male students in Western data and are more consistent with stereotypes of masculine social roles and aggressive behaviors. Unlike samples in Western countries where cutting and scratching are more prevalent among females and punching, burning, and self-hitting are more prevalent among male students (Andover et al., 2010; Whitlock et al., 2006, 2011), there were no gender differences in terms of the NSSI methods used in our sample. The lack of gender difference in the current study challenges the Western notion of typical self-cutters being female (also see Chandler, Myers, \& Platt, 2011) and is more consistent with most university studies in non-Western countries, where no gender differences were found in either prevalence rates of NSSI or methods used (Gholamrezaei et al., 2015).

Unlike results of studies conducted in the West (Plener et al., 2015), our findings showed no link between depression and lifetime NSSI prevalence across gender in the 
final models. This result can be interpreted as being consistent with the studies suggesting that the relationship between a history of psychiatric disorders and suicide might be weaker in Iran compared to the Western data (Panaghi et al., 2010). Toprak, Cetin, Guven, Can and Demircan (2011) suggest that, compared to the West, economic stressors and relationship conflicts are common risk factors for suicide in a Turkish context. Furthermore, Kleinman (1977) has noted that culture forms the expression and symptoms of psychological issues such as depression. Thus, the lack of association between depressive symp- toms and lifetime NSSI in our sample could be because our employed measure did not tap into cul- ture-specific symptoms of depression. In short, longitudinal studies with culture-specific instruments are needed to confirm and interpret the results.

Research indicates that deficits in regulating emotions are one of the most salient factors strongly linked to engaging in NSSI (Andover \& Morris, 2014). In Western studies that used the DERS, limited access to emotion regulation strategies is the dimension that is uniquely linked to engagement in NSSI (Andover \& Morris, 2014). The results of our study indicated simple correlations between goals, strategies and impulse subscales and lifetime NSSI among male students. Interestingly, all the correlated DERS subscales are reflective of the behavioral aspect of emotion dysregulation. In other words, goals, impulse and strategies subscales indicate difficulties controlling undesired behaviors and accessing desired behaviors when emotions are present rather than having difficulties experiencing and modulating inner emotional states (e.g., the aware and nonacceptance subscales) (Gratz \& Roemer, 2004). However, after controlling for anxiety and suicide ideation, none of the correlated DERS subscales remained significant for male students. This finding is contrary to the studies conducted in the West indicating a 
strong link between emotion dysregulation and NSSI. For instance, Gratz and Chapman (2007) found emotion dysregulation to be a strong risk factor of frequent NSSI among male undergraduate students. Interestingly, lifetime NSSI was associated with anxiety and suicide ideation for male students, which suggests some levels of undesirable emotional and cognitive states; however, lifetime engagement in NSSI was not linked to the male participants' difficulties in regulating those undesirable emotional/cognitive states. This result is consistent with the non-Western studies, which portray an unclear association between NSSI and emotion dysregulation (Gholamrezaei et al., 2015).

For female students, besides suicide ideation, the aware subscale remained the only significant predictor. The aware subscale indicates a 'lack of emotional awareness which consists of items reflecting the tendency to attend to and acknowledge emotions' (Gratz \& Roemer, 2004, p. 47) and these items are reverse coded, meaning that higher scores indicate less emotional awareness. According to the results, being more aware of one's own emotional experience significantly predicted a history of NSSI after controlling for psychological symptoms among the female students. To interpret this result, we may need to take a step back and look at the whole picture regarding the profile of self-injury behaviors among the female students. First, punching a wall and self-hitting were the most frequently reported methods for the female students. Punching and hitting can resemble outwardly focused aggressive behaviors. Second, results indicate that more than half of the female students reported self-injuring in the presence of others rather than when alone. Third, neither depressive symptoms assessed by the DASS (such as hopelessness, low mood, low self-worth and emptiness) nor anxiety symptoms (such as worry and restlessness) were predictors of NSSI, not even before entering the DERS 
subscales in the model. In short, the portrayed profile of females' NSSI in this study seems quite different from the typical descriptions of females' NSSI in the West (e.g., Bresin \& Schoenleber, 2015; Plener et al., 2015). Moreover, as noted earlier, some studies investigating suicidal behaviors in Iranian samples also revealed that compared with Western data, people who attempt suicide in Iran are less likely to have a history of psychological disorders and more likely to have been recently assaulted physically or verbally (Panaghi et al., 2010; Sheikhole- slami et al., 2008). Furthermore, it can be hypothesized that NSSI in Iran might also be linked to social and interpersonal factors rather than depression and anxiety. Further research could be useful to investigate whether helplessness, frustration or anger are present and whether they are regulated by self-injury in this context. In that case, higher levels of attending to the emotional experience of helplessness, frustration or anger might make students prone to self-injury behaviors such as punching a wall and self-hitting. Additionally, the sociocultural contexts of the sample such as gender role conflicts experienced by women (i.e., traditional versus modern gender roles) and the restrictions placed on women in Iran at both societal and family levels need to be taken into account (Tohidi, 1994).

The current study found a strong relationship between NSSI and SA. For both male and female students, having a history of NSSI predicted lifetime SA after controlling for depressive symptoms and suicidal ideations. In other words, NSSI maintained a relationship to SA even beyond suicide ideation and depression-related symptoms. This result is consistent with the research literature suggesting NSSI as a strong predictor of SA as it increases both the capacity and desire for suicide and decreases inhibition toward attempting suicide (Butler \& Malone, 2013; Whitlock et al., 
$\underline{2013})$.

\section{Strengths and limitations}

The present study must be interpreted in light of several limitations. First, a rather small sample size may have weakened the study's power. Second, no causal relationship can be inferred due to the cross-sectional nature of the study design. Third, the scale assessing NSSI was not yet validated in Iran making it vulnerable to potential bias. Fourth, retrospective self-report questionnaires were used in this study, which raises the possibility of response bias and also hinders context-sensitive interpretations of the results. Structured interviews as well as qualitative studies are needed to obtain more accurate data and facilitate understanding of NSSI within the sociocultural contexts of the population of interest. Fifth, in the current study a precise definition of NSSI that stipulates deliber- ate immediate tissue damage was employed; therefore, the current prevalence results are represen- tative of this type of NSSI, not of self-harm or self-injury that does not leave any indication of tissue damage (i.e., bleeding, bruising, marks). Finally, there might be some restrictions in the generalizabil- ity of the findings, as a convenience sampling method was used to recruit the participants. Overall, longitudinal studies with larger and more representative samples are needed to confirm the results of the present study.

On the positive side, our findings have the potential to suggest further pathways in researching NSSI among university students in Iran. Developmental trajectories of NSSI for male and female students can be investigated using longitudinal studies to clarify the role of emotion dysregulation and identification of culture-specific risk factors for NSSI. Another area of research could be an investigation of the level of practitioners' awareness 
about and comfort and training regarding working with students who engage in NSSI. Our findings suggest that self-injury behaviors are quite common among both male and female students in Iran. Considering the negative physical and psychological consequences of engaging in NSSI as well as the close relationship between NSSI and suicide attempt, it is very crucial to promote knowledge and awareness about NSSI among practitioners in Iran. Because of limited research on NSSI in Iran, practitioners might not take these behaviors seriously enough and interpret them as a form of attention seeking or manipulative behavior that needs to be ignored. In contrast, some practitioners might confuse self-injuring behaviors with an act of suicide and overreact to it, for example, by hospitalizing the individual or breaching the confidentiality, which can be shaming and stigmatizing. Further research could enhance the understanding of NSSI as a public health concern in the Iranian context and generate discussion and awareness among practitioners. Considering the young age of NSSI onset reported in the current study, early identification and intervention can be crucial.

\section{Conclusion}

This study found both commonalities and different patterns of NSSI and its risk factors compared to Western data. A lack of a gender difference in prevalence and methods used and a presence of out- ward-focused aggressive types of NSSI among female students were some points of divergence from the findings of studies conducted in Western countries. Moreover, inconsistent with Western data, emotion dysregulation was not a risk factor of lifetime NSSI across gender in our sample after con- trolling for psychological symptoms of anxiety, depression and suicide ideation. Consistent with Western data, NSSI is a strong predictor of SA even after controlling for depressive 
symptoms and suicidal ideation.

\section{References}

Adrian, M., Zeman, J., Erdley, C., Lisa, L., \& Sim, L. (2011). Emotional dysregulation and interpersonal difficulties as risk factors for nonsuicidal self-injury in adolescent girls. Journal of Abnormal Child Psychology, 39(3), 389-400. doi:10.1007/s10802-010-9465-3

American Psychiatric Association. (2013). Diagnostic and statistical manual of mental disorders (5th ed.). Washington, DC: Author.

Andover, M. S., \& Morris, B. W. (2014). Expanding and clarifying the role of emotion regulation in nonsuicidal self-injury. Canadian Journal of Psychiatry/Revue Canadienne de Psychiatrie, 59(11), 569-575. doi:10.1177/ 070674371405901102

Andover, M. S., Primack, J. M., Gibb, B. E., \& Pepper, C. M. (2010). An examination of nonsuicidal self-injury in men: Do men differ from women in basic NSSI characteristics? Archives of suicide research, 14(1), 79-88. doi:10.1080/ 13811110903479086

Baasher, T. (2001). Islam and mental health. Eastern Mediterranean Region Health Services Journal, 7, 372-376. 
Bayani, A. A. (2010). Reliability and preliminary evidence of validity of a farsi version of the depression anxiety stress scales 1, 2. Perceptual and Motor Skills, 111(1), 107-114. doi:10.2466/08.13.pms.111.4.107-114

Bentley, K. H., Nock, M. K., \& Barlow, D. H. (2014). The four-function model of nonsuicidal self-injury: Key directions for future research. Clinical Psychological Science, 2(5), 638656. doi: $10.1177 / 2167702613514563$

Bresin, K., \& Schoenleber, M. (2015). Gender differences in the prevalence of nonsuicidal selfinjury: A meta-analysis.

Clinical Psychology Review, 38, 55-64. doi:10.1016/j.cpr.2015.02.009

Butler, A. M., \& Malone, K. (2013). Attempted suicide v. non-suicidal self-injury: Behaviour, syndrome or diagnosis?

The British Journal of Psychiatry, 202(5), 324-325. doi:10.1192/bjp.bp.112.113506

Canino, G., Lewis-Fernandez, R., \& Bravo, M. (1997). Methodological challenges in crosscultural mental health research. Transcultural Psychiatry, 34(2), 163-184. doi:10.1177/136346159703400201

Chandler, A., Myers, F., \& Platt, S. (2011). The construction of self-injury in the clinical literature: A sociological exploration. Suicide and Life-Threatening Behavior, 41(1), 98109. doi:10.1111/j.1943-278x.2010.00003.x

Corcoran, P., \& Nagar, A. (2010). Suicide and marital status in Northern Ireland. Social Psychiatry and Psychiatric Epidemiology, 45(8), 795-800. doi:10.1007/s00127-009$\underline{0120-7}$

Favazza, A. R. (1996). Bodies under siege: Self-mutilation and body modification in culture and psychiatry. Baltimore: Johns Hopkins University Press. 
Gholamrezaei, M., De Stefano, J., \& Heath, N. L. (2015). Nonsuicidal self-injury across cultures and ethnic and racial minorities: A review. International Journal of Psychology. doi:10.1002/ijop.12230

Glenn, C. R., \& Klonsky, E. D. (2011). One-year test-retest reliability of the inventory of statements about self-injury (ISAS). Assessment. $\underline{\text { doi:1073191111411669 }}$

Gratz, K. L., \& Chapman, A. L. (2007). The role of emotional responding and childhood maltreatment in the devel- opment and maintenance of deliberate self-harm among male undergraduates. Psychology of Men \& Masculinity, 8(1), 1-14. doi:10.1037/1524$\underline{9220.8 .1 .1}$

Gratz, K. L., Conrad, S. D., \& Roemer, L. (2002). Risk factors for deliberate self-harm among college students.

American Journal of Orthopsychiatry, 72(1), 128-140. doi:10.1037//0002-9432.72.1.128

Gratz, K. L., \& Roemer, L. (2004). Multidimensional assessment of emotion regulation and dysregulation: Development, factor structure, and initial validation of the difficulties in emotion regulation scale. Journal of Psychopathology and Behavioral Assessment, 26(1), 41-54. doi:10.1023/b:joba.0000007455.08539.94

Gratz, K. L., \& Roemer, L. (2008). The relationship between emotion dysregulation and deliberate self-harm among female undergraduate students at an urban commuter university. Cognitive Behaviour Therapy, 37(1), 14-25. doi:10.1080/16506070701819524

Gutierrez, P. M., Osman, A., Barrios, F. X., \& Kopper, B. A. (2001). Development and initial validation of the self-harm behavior questionnaire. Journal of Personality Assessment, 77(3), 475-490. doi:10.1207/s15327752jpa7703_08 
Heath, N. L., Toste, J. R., Nedecheva, T., \& Charlebois, A. (2008). An examination of nonsuicidal self-injury among college students. Journal of Mental Health Counseling, 30(2), 137-156. doi:10.17744/mehc.30.2.8p879p3443514678 Henry, J. D., \& Crawford, J. R. (2005). The short-form version of the depression anxiety stress scales (DASS-21): Construct validity and normative data in a large non-clinical sample. British Journal of Clinical Psychology,

44(2), 227-239. doi:10.1348/014466505x29657

Khanzadeh, M., Saeediyan, M., Hosseinchari, M., \& Edrissi, F. (2012). Factor structure and psychometric properties of difficulties in emotional regulation scale. International Journal of Behavioral Sciences, 6(1), 87-96. Retrieved from http://journals.bmsu.ac.ir/jbs/index.php/jbs/article/viewFile/312/243

Kleinman, A. M. (1977). Depression, somatization and the "new cross-cultural psychiatry". Social Science \& Medicine (1967), 11(1), 3-9. doi:10.1016/0037-7856(77)90138-X

Klonsky, E. D. (2007). The functions of deliberate self-injury: A review of the evidence. Clinical psychology review, 27(2), 226-239. doi:10.1016/j.cpr.2006.08.002

Klonsky, E. D., \& Glenn, C. R. (2009). Assessing the functions of non-suicidal self-injury: Psychometric properties of the inventory of statements about self-injury (ISAS). Journal of Psychopathology and Behavioral Assessment, 31(3), 215-219. doi:10.1007/s10862$\underline{008-9107-z}$

Klonsky, E. D., May, A. M., \& Glenn, C. R. (2013). The relationship between nonsuicidal selfinjury and attempted suicide: Converging evidence from four samples. Journal of Abnormal Psychology, 122(1), 231-237. doi:10.1037/ a0030278

Klonsky, E. D., \& Olino, T. M. (2008). Identifying clinically distinct subgroups of self-injurers 
among young adults: A latent class analysis. Journal of Consulting and Clinical Psychology, 76(1), 22-27. doi:10.1037/0022-006x.76.1.22 Kposowa, A. J. (2000). Marital status and suicide in the national longitudinal mortality study. Journal of Epidemiology

and Community Health, 54(4), 254-261. doi:10.1136/jech.54.4.254

Lovibond, P. F., \& Lovibond, S. H. (1995). The structure of negative emotional states: Comparison of the depression anxiety stress scales (DASS) with the beck depression and anxiety inventories. Behaviour Research and Therapy, 33(3), 335-343. doi:10.1016/0005-7967(94)00075-u

Moran, P., Coffey, C., Romaniuk, H., Olsson, C., Borschmann, R., Carlin, J. B., \& Patton, G. C. (2012). The natural history of self-harm from adolescence to young adulthood: A population-based cohort study. The Lancet, 379 (9812), 236-243. doi:10.1016/S0140$\underline{6736(11) 61141-0}$

Muehlenkamp, J. J., Claes, L., Havertape, L., \& Plener, P. L. (2012). International prevalence of adolescent non-suicidal self-injury and deliberate self-harm. Child and Adolescent Psychiatry and Mental Health, 6(10), 1-9. doi:10.1186/ 1753-2000-6-10

Nixon, M. K., \& Heath, N. L. (2009). Self-injury in youth: The essential guide to assessment and intervention. New York: Routledge.

Nock, M. K. (2010). Self-injury. Annual Review of Clinical Psychology, 6, 339-363. doi:10.1146/annurev.clinpsy.

\section{$\underline{121208.131258}$}

Osman, A., Bagge, C. L., Gutierrez, P. M., Konick, L. C., Kopper, B. A., \& Barrios, F. X. (2001). The suicidal behaviors questionnaire-revised (SBQ-R): Validation with clinical 
and nonclinical samples. Assessment, 8(4), 443-454. doi:10. 1177/107319110100800409

Panaghi, L., Ahmadabadi, Z., Peiravi, H., \& Zahra Abolmasoomi, F. (2010). Suicide trend in university students during 2003 to 2008. Iranian Journal of Psychiatry and Clinical Psychology, 16(2), 87-98. Retrieved from http://ijpcp.iums. ac.ir/article-1-1040-en.pdf

Panaghi, L., Malakouti, S. K., Shoushtari, M. H., Movaghar, A. R., Yousefi-Nooraie, R., \& Abarashi, Z. (2007). Prevalence of self-inflicted burn and the related factors in Iran: A systematic review. Iranian Journal of Psychiatry, 2(4), 174-180. Retrieved from http://s3.amazonaws.com/academia.edu.documents/45621842/Prevalence_of_selfinflicted_burn_and_th20160514-230121d6aqdx.pdf?AWSAccessKeyId=AKIAJ56TQJRTWSMTNPEA\&Expires= $\underline{1481235880 \& \text { Signature }=\text { CyzdoGorjVLG1iCl6uPWXBrdg2E\%3D\&response-content- }}$ disposition=inline $\% 3 \mathrm{~B} \% 20$ filename $\%$ 3DPrevalence_of_self-inflicted_burn_and_th.pdf

Plener, P. L., Schumacher, T. S., Munz, L. M., \& Groschwitz, R. C. (2015). The longitudinal course of non-suicidal self- injury and deliberate self-harm: A systematic review of the literature. Borderline Personality Disorder and Emotion Dysregulation, 2(2). doi:10.1186/s40479-014-0024-3

Ross, S., \& Heath, N. L. (2007). How I deal with stress questionnaire (HIDS) (Unpublished manuscript). McGill University, Montreal, Canada.

Safa, M., Boroujerdi, F. G., Talischi, F., \& Masjedi, M. R. (2014). Relationship of coping styles with suicidal behavior in hospitalized asthma and chronic obstructive pulmonary disease patients: Substance abusers versus non-substance abusers. Tanaffos, 13(3), 23-30. Retrieved from https://www.researchgate.net/publication/272840215_Relationship of_Coping_Styles_with_Suicidal_Behavior_in_Hospitalized_Asthma_and_Chronic_Obstructive 


\section{$\underline{\text { Pulmonary_Disease }} \underline{\text { Patients_Substance Abusers_versus_Non-_Substance_Abusers }}$}

Sheikholeslami, H., Kani, C., \& Ziaee, A. (2008). Attempted suicide among Iranian population. Suicide and Life- Threatening Behavior, 38(4), 456-466. doi:10.1521/suli.2008.38.4.456

Stack, S., \& Wasserman, I. (2009). Gender and suicide risk: The role of wound site. Suicide and Life-Threatening Behavior, 39(1), 13-20. doi:10.1521/suli.2009.39.1.13

Swannell, S. V., Martin, G. E., Page, A., Hasking, P., \& St John, N. J. (2014). Prevalence of nonsuicidal self-injury in nonclinical samples: Systematic review, meta-analysis and meta-regression. Suicide and Life-Threatening Behavior, 44(3), 273-303. doi:10.1111/sltb.12070

Tohidi, N. (1994). Modernity, Islamization, and women in Iran. In V. Moghadam (Ed.), Gender and national identity

(pp. 110-147). London: Zed Books.

Toprak, S., Cetin, I., Guven, T., Can, G., \& Demircan, C. (2011). Self-harm, suicidal ideation and suicide attempts among college students. Psychiatry Research, 187(1), 140-144. doi:10.1016/j.psychres.2010.09.009

Whitlock, J., Eckenrode, J., \& Silverman, D. (2006). Self-injurious behaviors in a college population. Pediatrics, 117(6), 1939-1948. doi:10.1542/peds.2005-2543

Whitlock, J., Muehlenkamp, J., Eckenrode, J., Purington, A., Baral Abrams, G., Barreira, P., \& Kress, V. (2013). Nonsuicidal self-injury as a gateway to suicide in young adults. Journal of Adolescent Health, 52(4), 486-492. doi:10.1016/j.jadohealth.2012.09.010

Whitlock, J., Muehlenkamp, J., Purington, A., Eckenrode, J., Barreira, P., Baral Abrams, G., ... Chin, C. (2011). Nonsuicidal self-injury in a college population: General trends and sex differences. Journal of American College Health, 59(8), 691-698. 
$\underline{\text { doi: } 10.1080 / 07448481.2010 .529626}$

Wilcox, H. C., Arria, A. M., Caldeira, K. M., Vincent, K. B., Pinchevsky, G. M., \& O’Grady, K. E. (2012). Longitudinal predictors of past-year non-suicidal self-injury and motives among college students. Psychological Medicine, 42(04), 717-726.

doi: $10.1017 / \mathrm{S} 0033291711001814$

You, J., Leung, F., \& Fu, K. (2012). Exploring the reciprocal relations between nonsuicidal selfinjury, negative emotions and relationship problems in Chinese adolescents: A longitudinal cross-lag study. Journal of Abnormal Child Psychology, 40(5), 829-836. doi:10.1007/s10802-011-9597-0 
This is an Accepted Manuscript of an article published by Taylor \& Francis in 'International Journal of Culture and Mental Health' on 2016-12-20, available online: https://www.tandfonline.com/10.1080/17542863.2016.1265999.

\section{Tables and Figures}

Table 1. Demographic characteristics of study population $(n=554)$.

\begin{tabular}{lc}
\hline Characteristic & Percent $(n)$ \\
\hline Gender & \\
& $57.2(317)$ \\
Age range & $42.8(237)$ \\
3 & \\
& $61.1(339)$ \\
an 30 & $21.8(121)$ \\
& $10.8(60)$ \\
Level of education & $4.8(26)$ \\
Ir certificate & $1.5(8)$ \\
r's & \\
i & $2(11)$ \\
& $82.9(460)$ \\
Major & $8.8(49)$ \\
j & $6.1(33)$ \\
ring & $.2(1)$ \\
Language/Literature & \\
ies & $3.1(17)$ \\
Jgy/Education & $3.4(19)$ \\
dical & $13.3(74)$ \\
!ment/Accounting & $38.5(213)$ \\
Science & $9.4(51)$ \\
& $.5(3)$ \\
& $19.8(110)$ \\
\hline
\end{tabular}

Table2. Correlations between Depression Anxiety Stress Scale and Difficulties in EmotionRegulation Scalesubscales and lifetime non-suicidal self-injury.

\begin{tabular}{|c|c|c|}
\hline & Males & Females \\
\hline $\begin{array}{l}\text { symptoms } \\
\text { ation } \\
\text { in Emotion Regulation Scale } \\
\text { sptance }\end{array}$ & $\begin{array}{l}.078 \\
.171^{\star \star} \\
.109 \\
.247^{\star \star *} \\
.07 \\
.144^{\star} \\
.217^{\star \star *} \\
.04 \\
.133^{\star} \\
.114\end{array}$ & $\begin{array}{l}.167^{\star \star \star} \\
.225^{\star \star \star} \\
.191^{\star \star \star} \\
.319^{\star \star \star} \\
.114^{\star} \\
.07 \\
.157^{\star \star} \\
-.128^{\star} \\
.218^{\star \star \star} \\
.002\end{array}$ \\
\hline
\end{tabular}

Note: Point-biserial correlations with a Bonferroni correction of $p=.005$.

${ }^{*} p<.05$.

${ }^{* *} p<.01$.

${ }^{* * *} p<.005$.

Table 3. Correlations between non-suicidal self-injury, suicidal ideation, depressive symptoms, and lifetime suicide attempts.

\begin{tabular}{lll}
\hline & Males & Females \\
\hline deation & $.358^{* *}$ & $.236^{* *}$ \\
iidal self-injury & $.27^{* *}$ & $.317^{* *}$ \\
ive symptoms & $.18^{\star *}$ & $.151^{* *}$
\end{tabular}

Note:Point-biserial correlations are presented for depressive symptoms, suicidal ideation and suicide attempts, and phi coefficient is presented for non-suicidal self-injury and suicide attempts.

${ }^{* *}$ Correlations are significant at $p<.01$. 
Table 4. Rates of non-suicidal self-injury incidence and methods used by gender.

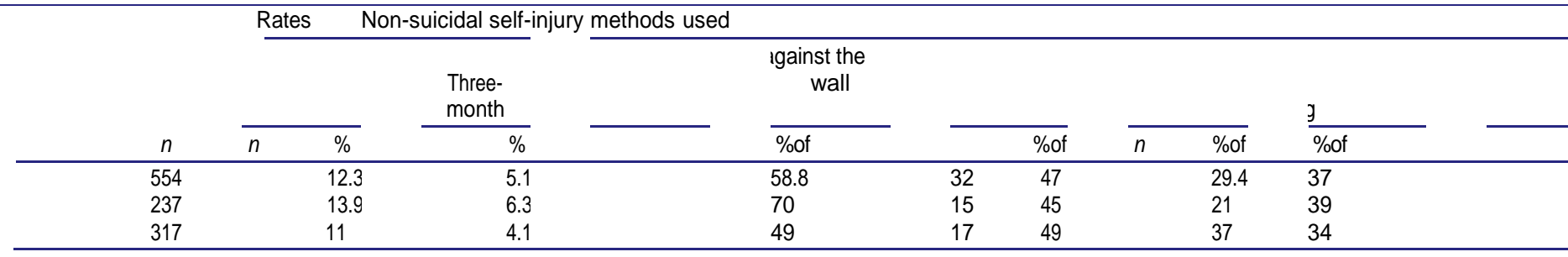

Table 5. Hierarchical logistic regression predicting lifetime non-suicidal self-injury.

\begin{tabular}{|c|c|c|c|c|c|c|c|c|c|}
\hline \multirow[b]{2}{*}{ Predictors } & \multicolumn{2}{|c|}{ Iales } & \multicolumn{3}{|c|}{ s } & \multicolumn{3}{|c|}{ Males } & \multirow[t]{2}{*}{ is } \\
\hline & $S E$ & $\overline{\text { Wald }}$ & $B$ & $S E$ & Wald & & $S E$ & Wald & \\
\hline Anxiety & 347 & $4.03^{*}$ & & 06 & & .056 & .054 & & .09 \\
\hline Depressive symptoms & & & & 05 & .002 & & & & .01 \\
\hline Suicide ideation & 16 & $9.31^{* *}$ & & 16 & $14.4^{\star \star}$ & & 17 & $7.07^{\star \star}$ & .59 \\
\hline Nonacceptance & & & & & & & & & -.03 \\
\hline Impulse & & & & & & .085 & 05 & & .01 \\
\hline Aware & & & & & & & & & -.11 \\
\hline Strategies & & & & & & & .045 & .147 & .03 \\
\hline Goals & & & & & & & 06 & & \\
\hline
\end{tabular}

${ }^{\star} p<.05,{ }^{* \star}<.01$.

Table 6. Hierarchical logistic regression predicting lifetime suicide attempts.

\begin{tabular}{|c|c|c|c|c|c|c|c|c|}
\hline & \multicolumn{4}{|c|}{ Block $1 \quad$ Block 2} & \multirow{2}{*}{\multicolumn{2}{|c|}{ es }} & \multirow{2}{*}{\multicolumn{2}{|c|}{ nales }} \\
\hline & es & & & & & & & \\
\hline & $S E$ & & Wald & & $S E$ & & $S E$ & $d$ \\
\hline $\begin{array}{l}\text { symptoms } \\
\text { :ation } \\
\text { al self-iniury }\end{array}$ & $\begin{array}{l}.05 \\
.83\end{array}$ & $\begin{array}{l}.07 \\
.24\end{array}$ & $\begin{array}{c}.45 \\
12.1^{* \star}\end{array}$ & $\begin{array}{l}.07 \\
.64\end{array}$ & $\begin{array}{r}.07 \\
.25 \\
1.67\end{array}$ & .71 & $\begin{array}{l}.05 \\
.24 \\
.66\end{array}$ & ** \\
\hline
\end{tabular}

${ }^{*} p<.01$. 